\title{
On Electronegativity, Hardness and Reactivity Descriptors: a new Property-Oriented basis set
}

Jesús Sánchez-Márquez, Victor García*, David Zorrilla and Manuel Fernández

Departamento de Química-Física, Facultad de Ciencias, Campus Universitario Río San

Pedro, Universidad de Cádiz, 11510 Puerto Real, Cádiz, Spain

*To whom correspondence should be addressed. E-mail: victorjesus.garcia@uca.es

\section{SUPPLEMENTARY MATERIAL}

\section{Table of contents:}

Table S1.- Pauling electronegativities ...................................................................... 3

Table S2.- Mulliken's electronegativities ................................................................... 3

Table S3.- Electronegativities in units of Mulliken ....................................................... 3

Table S4.- Allen electronegativities …………………………………………….... 3

Table S5.- Allen electronegativities ……………………......................................... 3

Table S6.- Rahm-Hoffmann electronegativities ............................................................... 4

Table S7.- Rahm-Hoffmann electronegativities ............................................................... 4

Table S8.- Relationships yi $=\mathrm{A} \cdot \mathrm{x}+\mathrm{B}$ of the Pauling, Allen and Rahm-Hoffmann

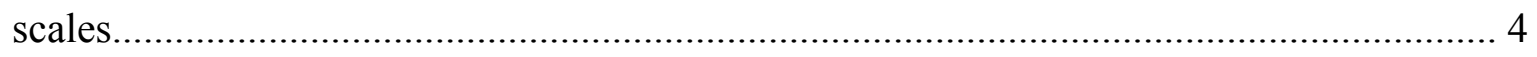

Table S9.- Atomic electrophilicities of the $\mathrm{X}-\mathrm{C} \equiv \mathrm{C}-\mathrm{H}$ molecules (where $\mathrm{X}=\mathrm{F}$ and $\mathrm{Cl}$ ) .... 4

Table S10.- Calculated electronegativities ................................................................. 4

Table S11.- Electronegativities calculated by adjusting polynomials ................................ 5

Table S12.- Hardnesses calculated by adjusting polynomials ........................................... 5

Table S13.- Hardnesses obtained with the extrapolation procedure ................................... 5 
Figure S1.- Relationship between experimental ionization energy and energy of the last

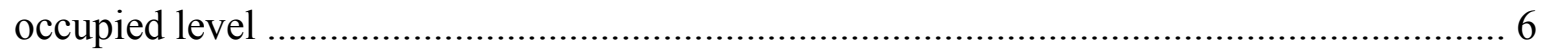

Figure S2.- Relationship between experimental ionization energy and the energy of the last

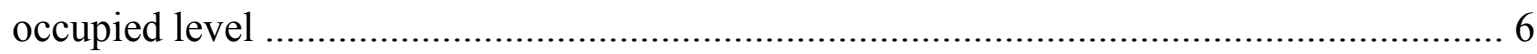

Figure S3.- Relationship between experimental ionization energy and the energy of the last

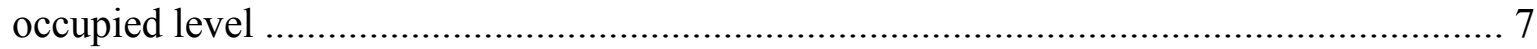

Figure S4.- Relationship between experimental ionization energy and the energy of the last

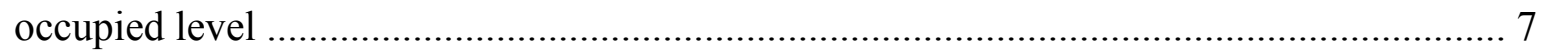

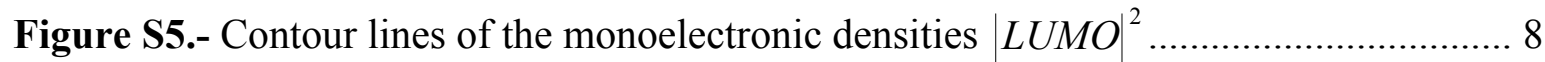


TABLES:

\begin{tabular}{|c|c|c|c|c|c|c|c|c|c|c|c|c|}
\hline $\mathrm{X}$ & $\mathrm{H}$ & $\mathrm{He}$ & $\mathrm{Li}$ & $\mathrm{Be}$ & $\mathrm{B}$ & $\mathrm{C}$ & $\mathrm{N}$ & $\mathrm{O}$ & $\mathrm{F}$ & $\mathrm{Ne}$ & $\mathrm{Na}$ & $\mathrm{Cl}$ \\
\hline $\mathbf{X}_{\mathrm{x}}$ & 2.1 & $\dot{\imath} ?$ & 1.0 & 1.5 & 2.0 & 2.5 & 3.0 & 3.5 & 4.0 & $\dot{\imath} ?$ & 0.9 & 3.0 \\
\hline
\end{tabular}

Table S1.- Pauling electronegativities (original values). Those of $\mathrm{He}$ or $\mathrm{Ne}$ could not be determined, because they did not form stable molecules.

\begin{tabular}{|c|c|c|c|c|c|c|c|c|c|c|c|c|}
\hline $\mathrm{X}$ & $\mathrm{H}$ & $\mathrm{He}$ & $\mathrm{Li}$ & $\mathrm{Be}$ & $\mathrm{B}$ & $\mathrm{C}$ & $\mathrm{N}$ & $\mathrm{O}$ & $\mathrm{F}$ & $\mathrm{Ne}$ & $\mathrm{Na}$ & $\mathrm{Cl}$ \\
\hline $\mathbf{I}_{\mathbf{X}}$ & 15.59 & 24.59 & 5.39 & 9.32 & 8.30 & 11.26 & 14.53 & 13.62 & 17.42 & 21.56 & 5.14 & 12.97 \\
\hline $\mathbf{A}_{\mathbf{X}}$ & 0.75 & $\approx 0$ & 0.62 & $\approx 0$ & 0.28 & 1.26 & $\approx 0$ & 1.46 & 3.40 & $\approx 0$ & 0.55 & 3.61 \\
\hline $\mathbf{1} / \mathbf{2}(\mathbf{I}+\mathrm{A})$ & 7.2 & 12.3 & 3.0 & 4.7 & 4.3 & 6.3 & 7.3 & 7.5 & 10.4 & 10.8 & 2.8 & 8.3 \\
\hline
\end{tabular}

Table S2.- Mulliken's electronegativities (in eV). Now those of noble gases can be estimated, as well as those of ions, molecules, excited states, etc.

\begin{tabular}{|c|c|c|c|c|c|c|c|c|c|c|c|c|}
\hline $\mathrm{X}$ & $\mathrm{H}$ & $\mathrm{He}$ & $\mathrm{Li}$ & $\mathrm{Be}$ & $\mathrm{B}$ & $\mathrm{C}$ & $\mathrm{N}$ & $\mathrm{O}$ & $\mathrm{F}$ & $\mathrm{Ne}$ & $\mathrm{Na}$ & $\mathrm{Cl}$ \\
\hline $\mathbf{X}_{\mathbf{x}}$ & 7.30 & 11.38 & 3.09 & 4.54 & 4.61 & 6.46 & 7.46 & 7.92 & 10.63 & 8.76 & 2.99 & 8.37 \\
\hline
\end{tabular}

Table S3.- Electronegativities calculated with B3LYP / Aug-cc-pV5Z, in units of Mulliken $(\mathrm{eV})$.

\begin{tabular}{|c|c|c|c|c|c|c|c|c|c|c|c|c|}
\hline $\mathrm{X}$ & $\mathrm{H}$ & $\mathrm{He}$ & $\mathrm{Li}$ & $\mathrm{Be}$ & $\mathrm{B}$ & $\mathrm{C}$ & $\mathrm{N}$ & $\mathrm{O}$ & $\mathrm{F}$ & $\mathrm{Ne}$ & $\mathrm{Na}$ & $\mathrm{Cl}$ \\
\hline $\boldsymbol{\chi}_{\text {Allen }}$ & 2.300 & --- & 0.912 & 1.576 & 2.051 & 2.544 & 3.066 & 3.610 & 4.193 & 4.787 & 0.869 & 2.869 \\
\hline
\end{tabular}

Table S4.- Allen electronegativities (arbitrary units) for the orbitals of some atoms. The units have been chosen so that the results are as similar as possible to those of the Pauling's scale.

\begin{tabular}{|c|c|c|c|c|c|c|c|c|c|c|c|c|}
\hline $\mathrm{X}$ & $\mathrm{H}$ & $\mathrm{He}$ & $\mathrm{Li}$ & $\mathrm{Be}$ & $\mathrm{B}$ & $\mathrm{C}$ & $\mathrm{N}$ & $\mathrm{O}$ & $\mathrm{F}$ & $\mathrm{Ne}$ & $\mathrm{Na}$ & $\mathrm{Cl}$ \\
\hline$\chi_{\text {Allen }}$ & -0.50 & --- & -0.20 & -0.31 & -0.40 & -0.51 & -0.63 & -0.77 & -0.94 & -1.12 & -0.18 & -0.65 \\
\hline
\end{tabular}

Table S5.- Allen electronegativities (atomic units) for the orbitals of some atoms, calculated using the HF / Aug-cc-pVQZ method. 


\begin{tabular}{|c|c|c|c|c|c|c|c|c|c|c|c|}
\hline $\mathrm{X}$ & $\mathrm{H}$ & $\mathrm{Li}$ & $\mathrm{Be}$ & $\mathrm{B}$ & $\mathrm{C}$ & $\mathrm{N}$ & $\mathrm{O}$ & $\mathrm{F}$ & $\mathrm{Ne}$ & $\mathrm{Na}$ & $\mathrm{Cl}$ \\
\hline $\mathbf{X}_{\text {Hoffmann }}$ & -0.50 & -1.72 & -2.52 & -3.34 & -4.16 & -4.98 & -5.80 & -6.62 & -7.45 & -8.71 & -16.71 \\
\hline
\end{tabular}

Table S6.- Rahm-Hoffmann electronegativities (atomic units) for the orbitals of some atoms, calculated using the HF/Aug-cc-pV5Z method.

\begin{tabular}{|c|c|c|c|c|c|c|c|c|c|c|c|}
\hline $\mathrm{X}$ & $\mathrm{H}$ & $\mathrm{Li}$ & $\mathrm{Be}$ & $\mathrm{B}$ & $\mathrm{C}$ & $\mathrm{N}$ & $\mathrm{O}$ & $\mathrm{F}$ & $\mathrm{Ne}$ & $\mathrm{Na}$ & $\mathrm{Cl}$ \\
\hline $\mathbf{X}_{\text {Hoffmann }}$ & -0.32 & -1.40 & -2.16 & -2.93 & -3.70 & -4.48 & -5.26 & -6.04 & -6.83 & -8.04 & -15.84 \\
\hline
\end{tabular}

Table S7.- Rahm-Hoffmann electronegativities (atomic units) for the orbitals of some atoms, calculated with the method B3LYP / Aug-cc-pV5Z.

\begin{tabular}{|l|c|c|c|}
\hline & A & B & $\mathbf{R}^{\mathbf{2}}$ \\
\hline Pauling & 0.4202 & -0.1113 & $\mathbf{0 . 9 2 4 5}$ \\
\hline Allen & 0.4501 & -0.2324 & $\mathbf{0 . 9 3 1 7}$ \\
\hline Rahm-Hoffmann & 0.6874 & -0.1088 & $\mathbf{0 . 9 2 4 6}$ \\
\hline
\end{tabular}

Table S8.- Relationships yi $=\mathrm{A} \cdot \mathrm{x}+\mathrm{B}$ of the Pauling, Allen and Rahm-Hoffmann scales with that of Mulliken

\begin{tabular}{|c|c|c|c|c|}
\hline $\mathbf{X}$ & $\mathbf{X}$ & $\mathbf{C 1}$ & $\mathbf{C 2}$ & $\mathbf{H}$ \\
\hline $\mathbf{X = F}$ & 0.0012 & 0.0035 & 0.0028 & 0.0141 \\
\hline $\mathbf{X = C l}$ & 0.0026 & 0.0019 & 0.0022 & 0.0143 \\
\hline
\end{tabular}

Table S9.- Atomic electrophilicities of the $\mathrm{X}-\mathrm{C} \equiv \mathrm{C}-\mathrm{H}$ molecules (where $\mathrm{X}=\mathrm{F}$ and $\mathrm{Cl}$ ) calculated with HF / Aug-cc-pV5Z.

\begin{tabular}{|c|c|c|c|c|c|c|c|c|c|c|c|c|}
\hline $\mathbf{X}$ & $\mathbf{B e}$ & $\mathbf{B}$ & $\mathbf{C}$ & $\mathbf{N}$ & $\mathbf{0}$ & $\mathbf{F}$ & $\mathbf{M g}$ & $\mathbf{A l}$ & $\mathbf{S i}$ & $\mathbf{P}$ & $\mathbf{S}$ & $\mathbf{C l}$ \\
\hline $\begin{array}{c}\mathbf{X} \\
\mathbf{( N = 2 )}\end{array}$ & 0.14 & 0.12 & 0.21 & 0.23 & 0.21 & 0.31 & 0.11 & 0.10 & 0.16 & 0.18 & 0.19 & 0.26 \\
\hline $\begin{array}{c}\mathbf{X} \\
(\mathbf{N}=\mathbf{1})\end{array}$ & 0.21 & 0.25 & 0.28 & 0.30 & 0.34 & 0.36 & 0.17 & 0.18 & 0.18 & 0.21 & 0.25 & 0.27 \\
\hline
\end{tabular}

Table S10.- Calculated electronegativities HF / Aug-cc-pV5Z and the formula (29). 


\begin{tabular}{|c|c|c|c|c|c|c|c|c|c|c|c|}
\hline Grade & Be & $\mathbf{B}$ & $\mathbf{C}$ & $\mathbf{N}$ & $\mathbf{O}$ & $\mathbf{F}$ & $\mathbf{A l}$ & $\mathbf{S i}$ & $\mathbf{P}$ & $\mathbf{S}$ & $\mathbf{C l}$ \\
\hline $4(|\mathrm{Q}| \leq 2)$ & 0.114 & 0.078 & 0.184 & 0.200 & 0.167 & 0.292 & 0.076 & 0.148 & 0.163 & 0.164 & 0.257 \\
\hline $2(|\mathrm{Q}| \leq 1)$ & 0.139 & 0.120 & 0.207 & 0.225 & 0.210 & 0.309 & 0.103 & 0.156 & 0.176 & 0.186 & 0.260 \\
\hline $\mathrm{Q} \rightarrow 0$ & 0.164 & 0.163 & 0.230 & 0.251 & 0.254 & 0.326 & 0.130 & 0.163 & 0.189 & 0.208 & 0.263 \\
\hline
\end{tabular}

Table S11. Electronegativities (a.u.) calculated by adjusting polynomials of degree 2 and 4 to the energies of the indicated atoms and their ions $(Q= \pm 1$ for grade $3,-Q= \pm 1 \pm 2$ for grade 4). The last row gives the linear extrapolation of the data obtained with $|\mathrm{Q}| \leq 1$ and $\mid$ $\mathrm{Q} \mid \leq 2$, to the case $\mathrm{Q} \rightarrow 0$.

\begin{tabular}{|c|c|c|c|c|c|c|c|c|c|c|c|}
\hline Grade & Be & $\mathbf{B}$ & $\mathbf{C}$ & $\mathbf{N}$ & $\mathbf{O}$ & $\mathbf{F}$ & $\mathbf{A l}$ & $\mathbf{S i}$ & $\mathbf{P}$ & $\mathbf{S}$ & $\mathbf{C l}$ \\
\hline $4(|\mathrm{Q}| \leq 2)$ & 0.328 & 0.357 & 0.385 & 0.598 & 0.445 & 0.529 & 0.194 & 0.248 & 0.395 & 0.298 & 0.339 \\
\hline $2(|\mathrm{Q}| \leq 1)$ & 0.313 & 0.350 & 0.380 & 0.571 & 0.463 & 0.532 & 0.206 & 0.249 & 0.376 & 0.307 & 0.345 \\
\hline $\mathrm{Q} \rightarrow 0$ & 0.298 & 0.344 & 0.376 & 0.544 & 0.481 & 0.535 & 0.217 & 0.250 & 0.357 & 0.316 & 0.351 \\
\hline
\end{tabular}

Table S12.- Hardnesses (a.u.) calculated by adjusting polynomials of degree 2 and 4 to the energies of the indicated atoms and their ions $(Q= \pm 1$ for grade $3,-Q= \pm 1 \pm 2$ for grade 4). The last row gives the linear extrapolation of the data obtained with $|\mathrm{Q}| \leq 1$ and $|\mathrm{Q}| \leq$ 2 , to the case $\mathrm{Q} \rightarrow 0$.

\begin{tabular}{|c|c|c|c|c|c|c|c|c|c|c|c|}
\hline & $\mathbf{B e}$ & $\mathbf{B}$ & $\mathbf{C}$ & $\mathbf{N}$ & $\mathbf{O}$ & $\mathbf{F}$ & $\mathbf{A l}$ & $\mathbf{S i}$ & $\mathbf{P}$ & $\mathbf{S}$ & $\mathbf{C l}$ \\
\hline $\mathbf{Q} \rightarrow \mathbf{0}$ & 0.298 & 0.344 & 0.376 & 0.544 & 0.481 & 0.535 & 0.217 & 0.250 & 0.357 & 0.316 & 0.351 \\
\hline Koopmans & 0.327 & 0.341 & 0.455 & 0.634 & 0.579 & 0.714 & 0.228 & 0.291 & 0.420 & 0.381 & 0.440 \\
\hline
\end{tabular}

Table S13.- Hardnesses (a.u.) obtained with the extrapolation procedure at $Q \rightarrow 0$, and obtained by applying the Koopmans approximation (HF method and Aug-cc-pV5Z base in both cases). 


\section{FIGURES:}

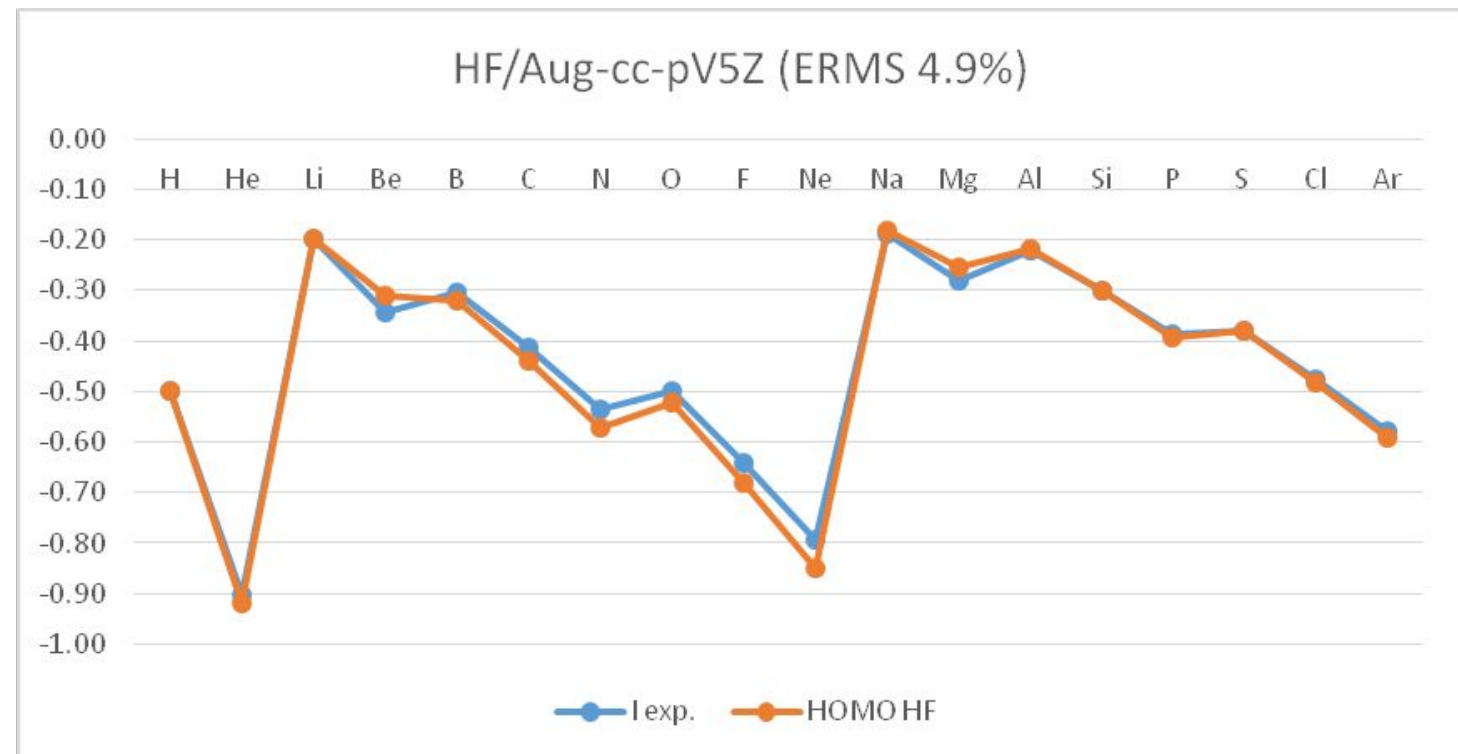

Figure S1.- Relationship between experimental ionization energy and energy of the last occupied level (HOAO), using Hartree-Fock (Aug-cc-pV5Z basis).

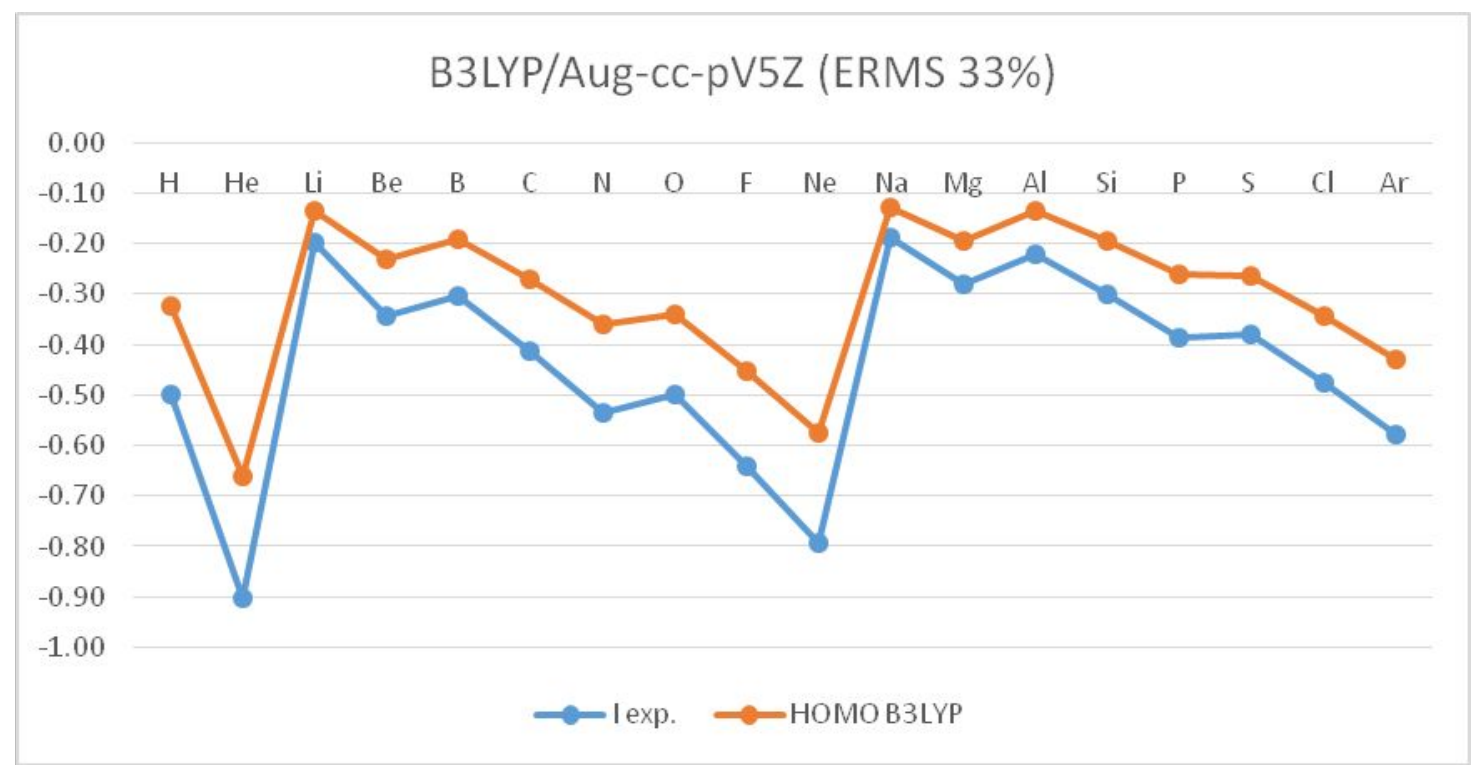

Figure S2.- Relationship between experimental ionization energy and the energy of the last occupied level (HOAO) using the standard DFT B3LYP. (Aug-cc-pV5Z basis). 


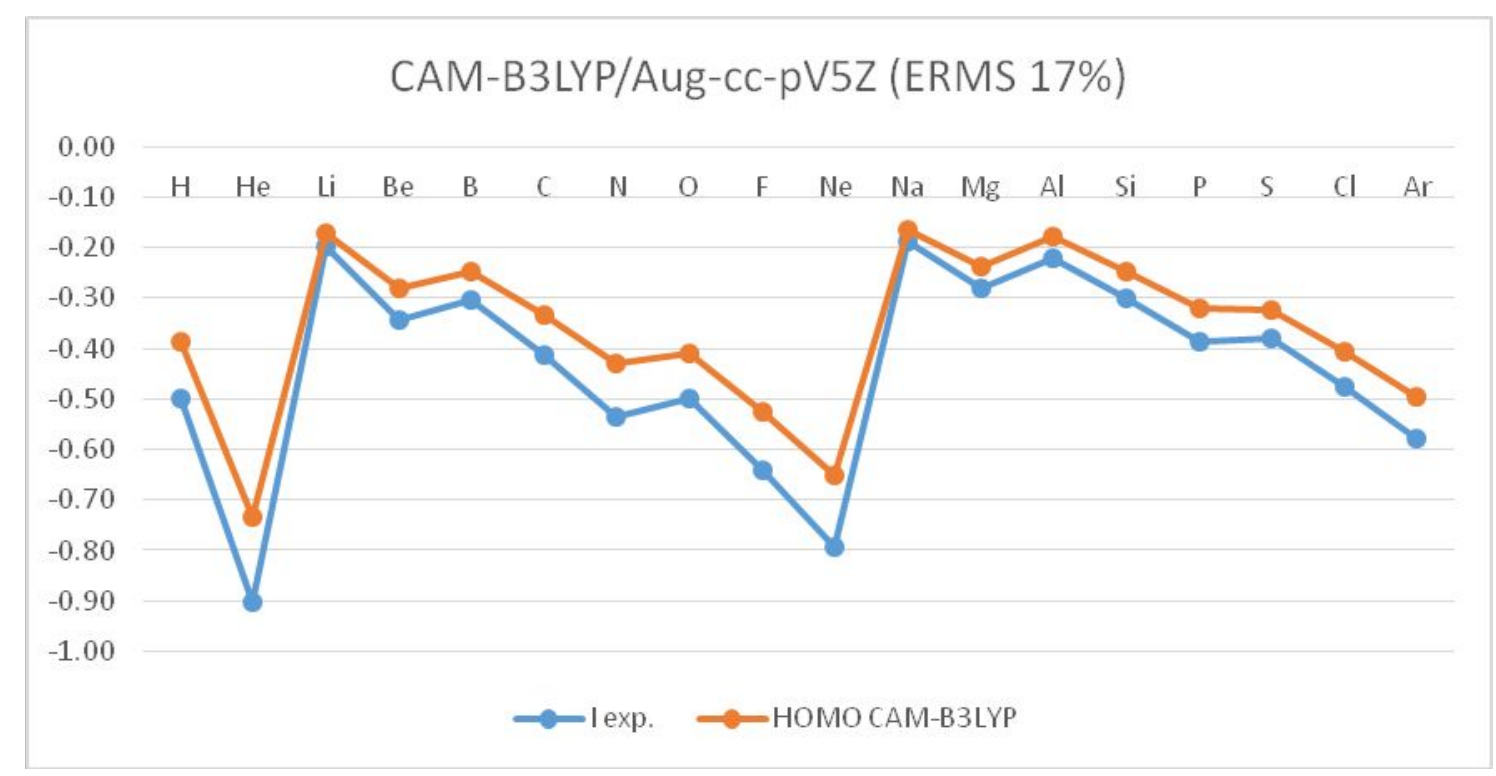

Figure S3.- Relationship between experimental ionization energy and the energy of the last occupied level (HOAO) using DFT with "Long Range Correction" CAM-B3LYP. (Aug-ccpV5Z basis).

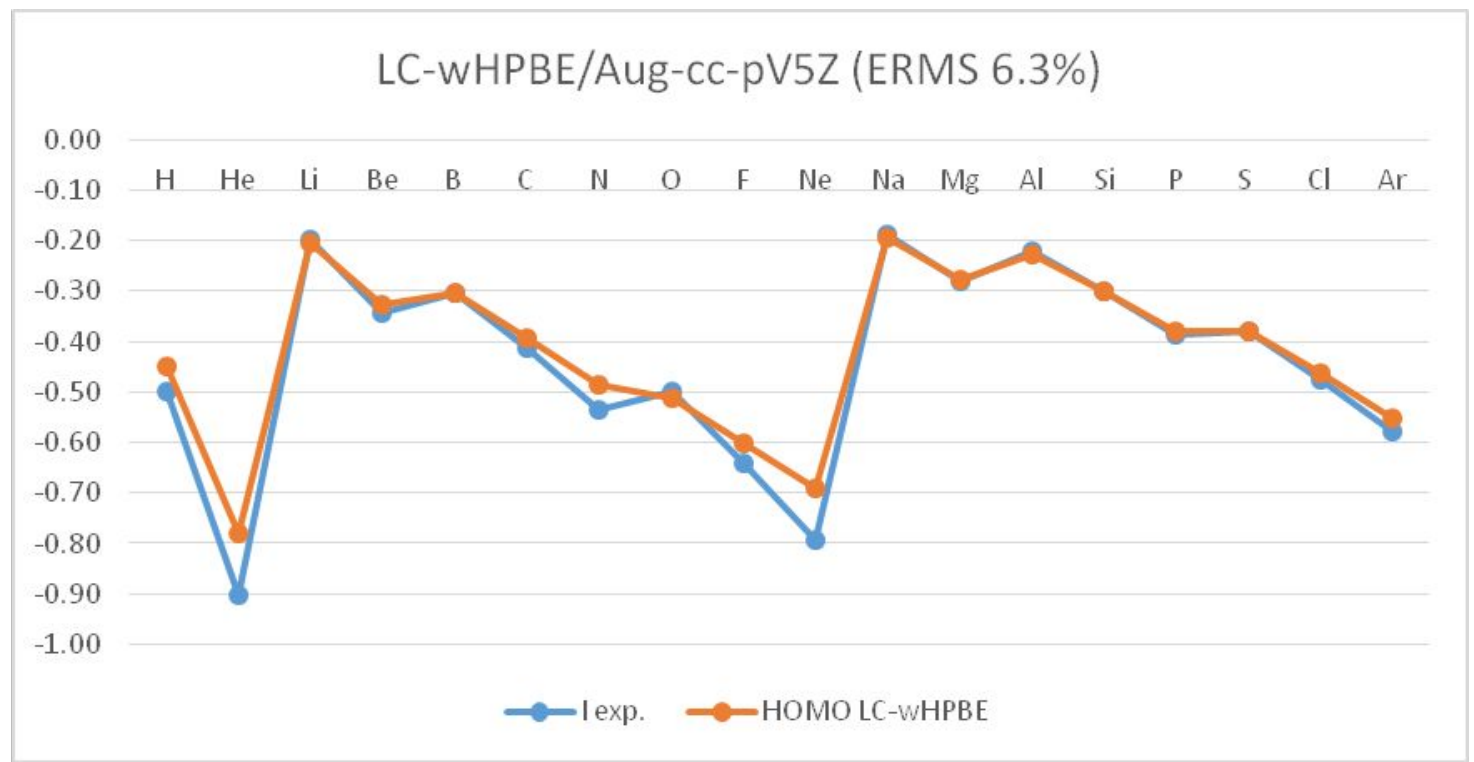

Figure S4.- Relationship between experimental ionization energy and the energy of the last occupied level (HOAO) using DFT with "Long Range Correction" LC-wHPBE. (Aug-ccpV5Z basis). 


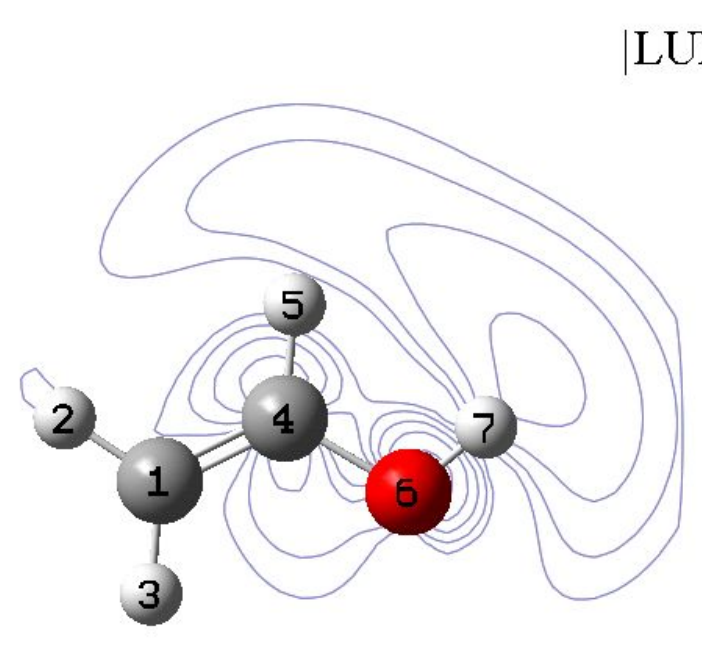

$6-311 G(d, p)$

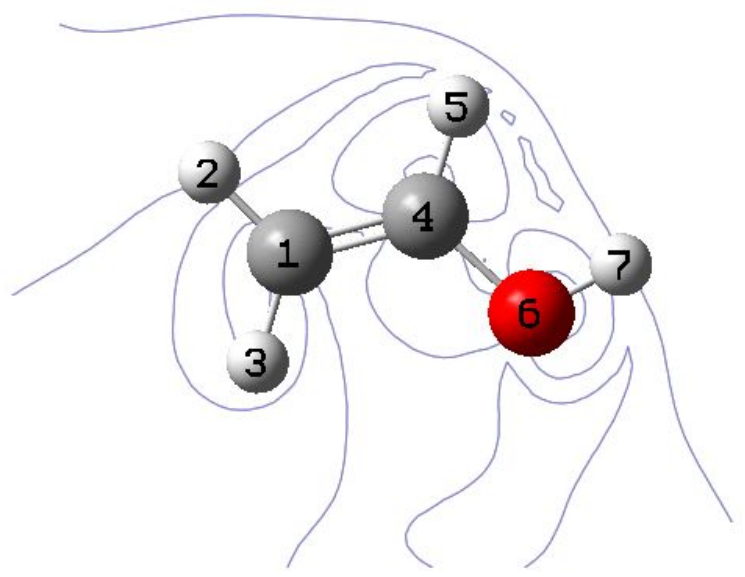

$6-311 \times x G(d, p)$

Figure S5.- Contour lines of the monoelectronic densities $|L U M O|^{2}$ calculated using the 6$311 \mathrm{G}(\mathrm{d}, \mathrm{p})$ and 6-311xxG(d,p) basis sets for the $\mathrm{CH}_{2} \mathrm{CHOH}$ molecule. 